\title{
Controlling attosecond electron dynamics by phase-stabilized polarization gating
}

\author{
I. J. SOLA ${ }^{1}$, E. MÉVEL ${ }^{1}$, L. ELOUGA 1 , E. CONSTANT ${ }^{1 *}$, V. STRELKOV ${ }^{2}$, L. POLETTO ${ }^{3}$, P. VILLORESI ${ }^{3}$, \\ E. BENEDETTI ${ }^{4}$, J.-P. CAUMES ${ }^{4}$, S. STAGIRA ${ }^{4}$, C. VOZZI ${ }^{4}$, G. SANSONE ${ }^{4}$ AND M. NISOLI ${ }^{4}$ \\ ${ }^{1}$ Centre Lasers Intenses et Applications, Université Bordeaux I, UMR 5107 (CNRS, Bordeaux 1, CEA), Domaine du Haut Carré, 351 Cours de la Libération, \\ 33405 Talence Cédex, France \\ ${ }^{2}$ General Physics Institute of Russian Academy of Sciences, 38 Vavilova st., Moscow 119991, Russia \\ ${ }^{3}$ CNR-INFM, Laboratory of Ultraviolet and X-ray Optical Research, Department of Information Engineering, University of Padova, Via Gradenigo, 6 , 35131 Padova, Italy \\ ${ }^{4}$ CNR-INFM, National Laboratory for Ultrafast and Ultraintense Optical Science, Department of Physics, Politecnico of Milan, Piazza L. da Vinci 32, 20133 Milano, Italy \\ *e-mail: constant@celia.u-bordeaux1.fr
}

1 ttosecond electron wavepackets are produced when an intense laser field ionizes an atom or a molecule ${ }^{1}$. When the laser field drives the wavepackets back to the parent ion, they interfere with the bound wavefunction, producing coherent subfemtosecond extreme-ultraviolet light bursts. When only a single return is possible $e^{2,3}$, an isolated attosecond pulse is generated. Here we demonstrate that by modulating the polarization of a carrier-envelope phase-stabilized short laser pulse $^{4}$, we can finely control the electron-wavepacket dynamics. We use high-order harmonic generation to probe these dynamics. Under optimized conditions, we observe the signature of a single return of the electron wavepacket over a large range of energies. This temporally confines the extreme-ultraviolet emission to an isolated attosecond pulse with a broad and tunable bandwidth. Our approach is very general, and extends the bandwidth of attosecond isolated pulses in such a way that pulses of a few attoseconds seem achievable. Similar temporal resolution could also be achieved by directly using the broadband electron wavepacket. This opens up a new regime for timeresolved tomography of atomic or molecular wavefunctions $s^{5,6}$ and ultrafast dynamics.

During high-order harmonic generation (HHG) in $\mathrm{gas}^{7}$, short electron wavepackets (EWPs) are periodically released by high-field ionization. Their subsequent coherent interaction with the remaining bound wavefunction leads to coherent extremeultraviolet (XUV) emission. The $T_{0} / 2$ periodicity of this process ( $T_{0}$ being the laser optical period) ensures that only odd harmonics of the fundamental radiation are emitted. Temporally, the XUV pulses are emitted as a train of chirped attosecond pulses ${ }^{8-10}$ ( 1 attosecond $=10^{-18} \mathrm{~s}$ ). For both plateau (low energy) and cut-off (high energy) harmonics, specific focusing conditions ensure that only a single attosecond pulse is emitted every half cycle ${ }^{11,12}$.

Extracting an isolated attosecond pulse from this train requires breaking the periodicity of the process, so that XUV emission is only possible within a single half cycle of the fundamental pulse. In this way, isolated 250-attosecond-long

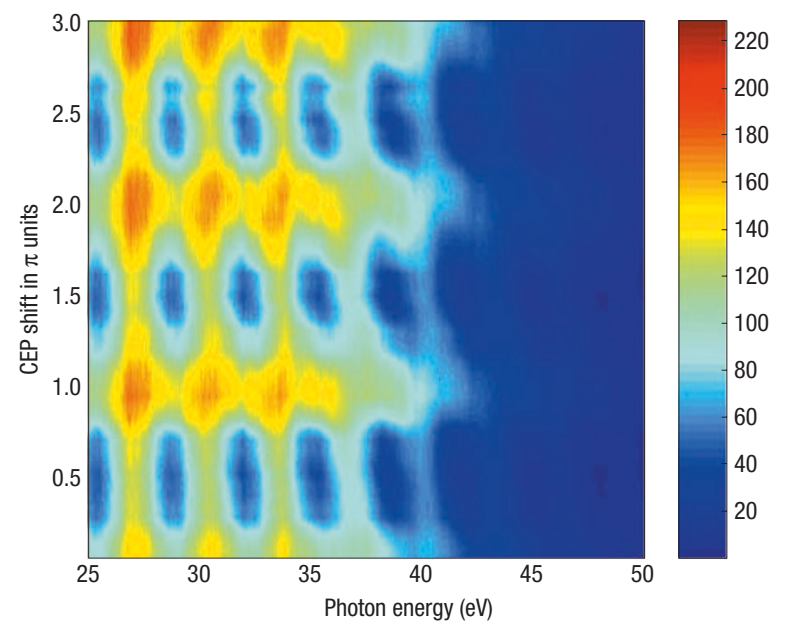

Figure 1 Spectra generated in argon. Spectra emitted from an argon medium irradiated with a polarization-modulated pulse ( $\tau=5 \mathrm{fs}, \delta=6.2 \mathrm{fs}, \beta=0^{\circ}$ ) as a function of the CEP shift. For some CEPs, harmonic peaks appear, whereas for other CEPs, they broaden up to a continuum.

pulses were recently obtained ${ }^{13}$ by selecting the (highly intensity dependent) cut-off harmonics generated in neon by a 5 -fs linearly polarized, fundamental pulse with stabilized carrierenvelope phase (CEP). With this technique, the minimum pulse duration achievable is limited by the $(\sim 10 \mathrm{eV})$ bandwidth of the selected cut-off harmonics, which prevents us reaching the sub-100-attosecond domain.

To isolate a broadband attosecond pulse, we used a different approach ${ }^{2}$. Our approach relies on the strong HHG sensitivity on the ellipticity, $\varepsilon$, of the fundamental field, which is largely 


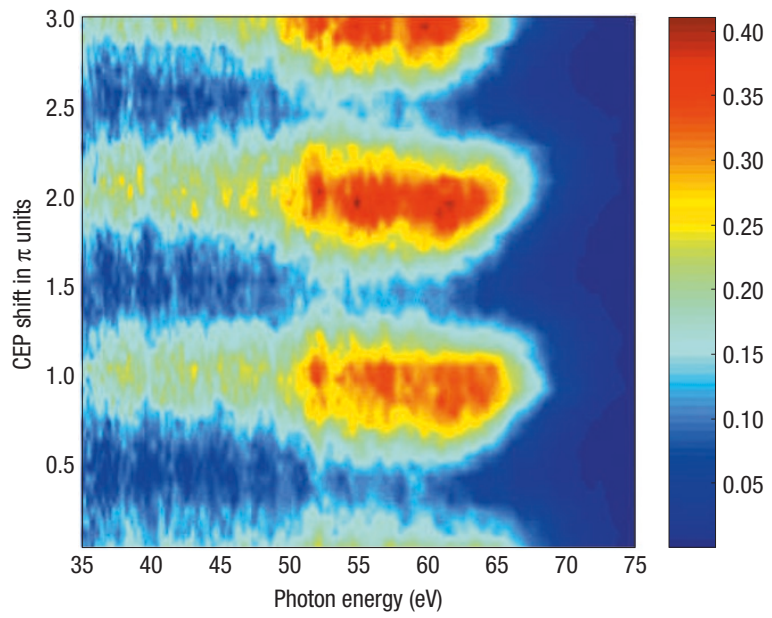

Figure 2 Spectra generated in neon. Spectra emitted from a neon medium irradiated with a polarization-modulated pulse ( $\tau=5 \mathrm{fs}, \delta=6.2 \mathrm{fs}, \beta=0^{\circ}$ ) as a function of the CEP shift. The efficiency of the emission process strongly depends on the CEP, and the spectra are continuous at maximum efficiency.

wavelength independent. The HHG efficiency is maximum when $\varepsilon=0$ (linear polarization) and decreases quickly as $\varepsilon$ increases. Carrying out HHG with a fundamental pulse that is only linearly polarized for a short time (and elliptically polarized elsewhere) ensures that the XUV emission is temporally confined ${ }^{2,14-21}$ inside a 'polarization gate', where the fundamental ellipticity is small.

To modulate the laser-pulse polarization, we developed a simple technique ${ }^{16}$ using birefringent plates (see the Methods section). The polarization-gate width ${ }^{22}, \tau_{\mathrm{g}}$, given by

$$
\tau_{\mathrm{g}}=\frac{\varepsilon_{\mathrm{thr}} \tau^{2}}{\ln (2) \delta|\cos (2 \beta)|}
$$

( $\varepsilon_{\mathrm{thr}}$ being a threshold ellipticity, $\tau$ the minimum driving-pulse duration) can be controlled through a delay $(\delta)$ and an angle $(\beta)$. For polarization gates as short as $T_{0} / 2$, required to produce isolated attosecond pulses, the $\mathrm{CEP}^{4}$ of the driving pulse also plays a crucial role in controlling the EWP dynamics ${ }^{19,20,22}$. The CEP, controlling the field position in the envelope, locates the possible recombination times (linked to the field) inside or outside the gate (linked to the envelope). Here, we use our robust polarization modulator in conjunction with CEP-stabilized 5-fs pulses to create a polarization gate shorter than $T_{0} / 2$. We observe the signature of the EWP dynamic control by recording the CEP dependence of the emitted XUV spectra.

Without polarization-induced confinement, we observed welldefined harmonics (we obtained 0.6-eV-wide plateau harmonics with both plates at $\alpha=\beta=45^{\circ}$ ) that were weakly affected by the CEP, except for the cut-off harmonics generated in neon ${ }^{23}$. When decreasing $\beta$, the interaction parameters were kept constant, except for the gate width, which was continuously decreased, and we observed a progressive broadening of the harmonics associated with a growing dependence on the CEP. With strong polarizationinduced confinement, the CEP influence was very strong on the whole spectrum. Figure 1 shows 50 XUV spectra (horizontal lines) generated in argon (with $\tau=5 \mathrm{fs}, \delta=6.2 \mathrm{fs}, \beta=0^{\circ}$ ) for 50 different CEP values varied over a $3 \pi$ range. The spectra amplitudes and shapes periodically change for a CEP variation $\Delta \psi=\pi$. For those CEP minimizing the XUV emission, clear harmonic
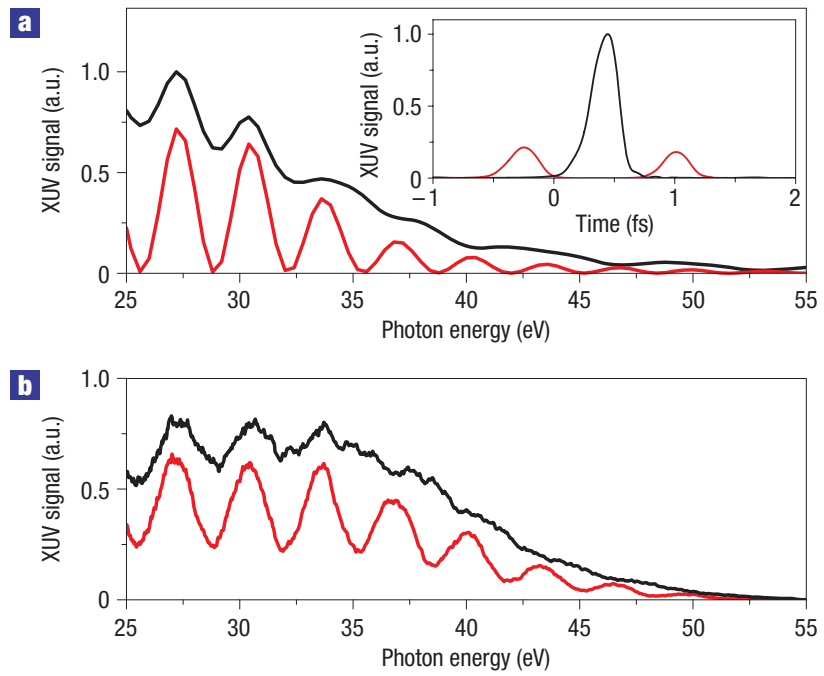

Figure 3 Comparison between simulated and experimental spectra.

$\mathbf{a}$, Simulated and $\mathbf{b}$, experimental spectra obtained for $\mathrm{HHG}$ in argon with a polarization-modulated pulse ( $\tau=5 \mathrm{fs}, \delta=6.2 \mathrm{fs}, \beta=0^{\circ}$ ) for two CEPs differing by $\pi / 2$. The inset shows the simulated temporal profile associated with these CEPS (considering all of the harmonics above the order of 11 emitted in the laser direction from a 1-mm-thick argon jet).

structures are present. After a $\pi / 2$ CEP shift, the efficiency becomes maximum, the harmonic structures vanish and the spectra become continuous in the range observed, with weak modulations for the lowest harmonics. For the cut-off part of the spectrum, the energy in the XUV continuum is estimated ${ }^{24}$ to be $\sim 20 \mathrm{pJ}$ per shot (efficiency: $10^{-7}$ ) and for the $25-50 \mathrm{eV}$ spectral range, the total XUV energy per shot is $\sim 70 \mathrm{pJ}$.

When generating XUV in neon (using the same polarization modulation but a higher intensity in the gas medium), a stronger CEP influence was observed (Fig. 2). The emission efficiency still evolves with the same $\pi$ periodicity but changes more strongly with the CEP. At maximum efficiency, the spectra were again continuous over all of the recorded bandwidths with a $30-\mathrm{eV}$ fullwidth at half-maximum, and the energy in the continuum was $\sim 1$ pJ (efficiency: $5 \times 10^{-9}$ ).

Controlling $\tau_{\mathrm{g}}$ by changing $\delta$ changed the XUV spectra dramatically. By reducing $\delta$ from 6.2 to 5 fs (to increase $\tau_{\mathrm{g}}$ ) and generating XUV in neon, we observed an increase in the generation efficiency and a CEP evolution of the XUV spectra similar to that displayed in Fig. 1 for argon with broad, but resolved, harmonics for some CEP values and broadband continuous spectra (reaching the $100 \mathrm{eV}$ photon energy, and with an integrated energy of $\sim 3 \mathrm{pJ}$ per shot) for other CEP values. Slightly larger gates induced a large CEP-dependent harmonic frequency shift (also apparent in Fig. 1 for the highest photon energy) that gives an apparent continuous spectrum without CEP stabilization. This clearly confirms that the observation of a continuous spectrum is only meaningful when the CEP is controlled ${ }^{4,19}$.

Decreasing $\tau_{\mathrm{g}}$ by increasing $\delta$ allowed us to generate XUV continuous spectra, in argon, that show the strong modulation in efficiency formerly described with neon (Fig. 2). Increasing $\delta$ reduced the intensity in the gate, and thus decreased the emission efficiency.

We also verified the importance of selecting a single trajectory ${ }^{11,12}$ by properly positioning the medium after the laser focus, as the confinement effect was maximum there. 
Table 1 Measured threshold ellipticity, $\varepsilon_{\text {thr }}$, for harmonic generation (order $q=13$ to 25 ) in argon and $(q=27$ to 73 ) in neon.

\begin{tabular}{|c|c|c|c|c|c|c|c|c|c|c|c|c|c|c|}
\hline$q$ & 13 & 15 & 17 & 19 & 21 & 23 & 25 & 27 & 31 & 35 & 41 & 49 & 53 & 73 \\
\hline$\varepsilon_{\text {thr }}(\%)$ & 17.5 & 16 & 14 & 14 & 12 & 12 & 12 & 11.5 & 11.5 & 11.5 & 11 & 10 & 10 & 8 \\
\hline
\end{tabular}

The strong CEP dependence of the spectra is consistent with a strong confinement of the XUV emission. For the highest harmonics generated in argon, either one or two XUV bursts, depending on the CEP, are emitted leading to either a continuous spectrum or a modulated spectrum with broad harmonic structures. The 1.6-eV width of the harmonics (Fig. 3b) is the signature of the interference between only two XUV bursts. For the lowest order harmonics obtained in argon (Fig. 3b), harmonic structures are always noticeable. This implies that these low harmonics are less confined (as expected from Table 1 because they are slightly less ellipticity sensitive) than the higher ones, and indicates that two pulses interfere. However, the observed modulation depth (10-20\% of the signal) can be produced by a second attosecond pulse when its energy is less than $1 \%$ of the main-pulse energy. Table 1 shows that the high harmonics generated in neon are more ellipticity sensitive than those generated in argon. Therefore, the polarization-induced confinement is stronger for XUV emission in neon than in argon, as observed here.

The experimental results were simulated by a numerical model $1^{14,20,22}$ derived from the three-steps model, including Coulomb attraction of the nucleus and propagation in the generating medium ( $1 \mathrm{~mm}$ long). In Fig. 3a, we show two simulated spectra generated in argon (with $\tau=5 \mathrm{fs}, \delta=6.2 \mathrm{fs}, \beta=0^{\circ}$ ) for two CEP values differing by $\pi / 2$. The corresponding experimental spectra are shown in Fig. 3b, and the overall agreement is striking. Only the fringe contrast is weaker experimentally than theoretically. This can be due to the fact that only on-axis emission is simulated (whereas the experimental spectra correspond to the XUV emission in all directions) and/or to the small $(0.1 \mathrm{rad})$ CEP fluctuations. After selecting all of the harmonics with orders higher than 11 (experimentally this selection is possible with an Al filter), this analysis gives us the temporal shape of the XUV pulses (Fig. 3a inset). Changing the CEP leads to a situation where either two XUV attosecond pulses or a single attosecond burst (with a prepulse of energy equal to $0.5 \%$ of the main-pulse energy), which has a duration of 260 attoseconds (165 attoseconds after chirp compensation), are emitted. The simulations are also in good agreement with the results obtained in neon and show that, in the spectral range observed, chirped isolated pulses of 205 attoseconds (85 attoseconds after chirp compensation) can be produced over a broad range of CEP in the experimental conditions of Fig. 2 (with very good contrast). Therefore, after chirp compensation, sub-100-attosecond XUV pulses are accessible with this technique. Chirp compensation was recently demonstrated ${ }^{25}$ in the wavelength range corresponding to HHG in argon, and techniques have been published for other spectral domains ${ }^{26,27}$.

The generation of such broadband isolated EWP and XUV pulses opens the way for new applications in attoscience. Our approach enables us to vary the carrier wavelength of an isolated attosecond pulse, and therefore allows selective studies. For instance, at moderate XUV energy, isolated attosecond pulses can now be produced, and are well suited to follow ultrafast processes, such as proton transfer or evolution of highly excited molecular states. At higher energy, sub-100-attosecond pulses are within reach, and have the required duration to study electron-electron interactions, such as Auger processes or electron correlation. Trains of short EWPs were also directly used to carry out tomography of stationary molecular orbitals ${ }^{5}$. Broadband, isolated short EWPs can be used to carry out time-resolved tomography of non- stationary molecular orbitals, or even to probe the ultrafast temporal evolution of bound EWPs ${ }^{6}$.

The energy of the isolated XUV pulses emitted through polarization gating can be increased in several ways. Here the generation parameters (gas pressure, interaction geometry, laser intensity) were chosen to unambiguously observe the confinement, and were probably not optimal for maximum efficiency. Higherenergy few-cycle pulses will also be available soon (through optical parametric chirped pulse amplification, OPCA, for instance) and can directly be used with this technique. Alternative polarizationgating techniques ${ }^{2,15,28}$ also exist, and could allow the use of longer fundamental pulses with higher energy. Finally, polarization gates shorter than $T_{0} / 2$ are required here for the production of isolated attosecond pulses only because the periodicity of HHG is $T_{0} / 2$. Adding a weak second-harmonic field to the fundamental pulse can increase this periodicity to $T_{0}$, in which case larger gates can be used which can be created with even higher-energy, longer fundamental pulses.

The XUV confinement to an isolated attosecond burst by polarization gating is possible both in the plateau and cut-off region because these harmonics evolve similarly with $\varepsilon$. This ellipticity dependence is very general, and we have shown that the central frequency of the attosecond pulse can be tuned by changing the gas. Other media can be used to further increase the bandwidth and tunability of isolated attosecond pulses. This bandwidth is limited by the cut-off energy, which has reached the $\mathrm{keV} \mathrm{level}{ }^{29}$ and can still be extended. Isolated pulses with a several-hundred-eV bandwidth, and a duration of a few attoseconds could then be obtained after chirp compensation, and will allow us to study ultrafast multiple-electron rearrangement. Using isolated broadband EWP, similar temporal resolution would be achievable for high-spatialresolution tomography of electronic wavefunctions.

\section{METHODS}

The polarization-gating technique that we have developed ${ }^{16}$ provides both a rapid modulation of the ellipticity and a $100 \%$ transmission of the input pulse energy. It involves transmitting an incoming pulse through two birefringent (quartz) plates: a first thick plate and a zero-order quarter waveplate. The incoming pulse is linearly polarized (along a direction that defines the reference for the angles $\alpha$ and $\beta$ ) at $\alpha=45^{\circ}$ of the neutral axis of the first thick plate. By crossing it, the pulse is split into two delayed (delay $\delta$ ) cross-polarized twin pulses. The delay, $\delta$, is proportional to the plate thickness, and depends on the plate indexes at the carrier frequency of the pulse. Several thicknesses have been used to change $\delta$ (a 193- $\mu \mathrm{m}$ thickness induced $\delta=6.2 \mathrm{fs}$ at the carrier wavelength of $750 \mathrm{~nm}$ ). Alternatively, we also replaced this plate with two mobile quartz wedges (birefringent plate with controllable thickness) to continuously control $\delta$. When necessary, we could tune the plate thickness to obtain a quarter waveplate at the pulse carrier wavelength. In that case, the outgoing pulse polarization evolves from linear to circular, and back to linear. After crossing a second zero-order broadband quarter waveplate (with its neutral axis at an angle $\beta$ with respect to the reference direction), the outgoing pulse is linearly polarized at its centre and circularly polarized (for $\beta=0^{\circ}$ ) in its wings. Therefore, it has a time-dependent polarization, suitable for confining HHG. The polarization modulation is defined by $\delta$, and by the minimum driving-pulse duration $\tau$ (we only consider pulses that are Fourier-limited in the interaction region). By considering gaussian pulses and defining the threshold ellipticity (see Table 1), $\varepsilon_{\mathrm{thr}}$, as the constant ellipticity for which the harmonic signal is decreased to $50 \%$ of the maximum signal (obtained for $\varepsilon=0)$, the polarization gate has a width given by ${ }^{22} \tau_{\mathrm{g}}=\varepsilon_{\mathrm{thr}} \tau^{2} /(\ln (2) \delta)$. 
Isolating a single attosecond pulse requires $\tau_{\mathrm{g}}<T_{0} / 2$. When the first quartz waveplate is a quarter waveplate, the gate width can be controlled further ${ }^{16}$ by $\beta$ without changing the intensity profile of the outgoing pulse. Larger gate widths, given by equation (1) can then be obtained.

As HHG is a highly nonlinear process, it is important to keep the highest intensity inside the gate to maximize the efficiency. Ideally, the peak intensity of the pulse must be reached in the gate, which implies $\delta \leq \tau$. When delays larger than $\tau$ are used, the intensity shows a minimum inside the gate, and the polarization confinement can be influenced by a pre-pulse of higher intensity. Combining $\delta \leq \tau$ with $\tau_{\mathrm{g}} \sim T_{0} / 2$ requires pulses shorter than $6-7$ fs to efficiently generate isolated attosecond pulses.

The 5 -fs pulses are produced by compression ${ }^{30}$ of 25 -fs light pulses generated by a Ti:sapphire laser system $(0.7-\mathrm{mJ}$ energy, $1-\mathrm{kHz}$ repetition rate). Such compression involves spectral broadening of the pulses inside an argon-filled tapered hollow fibre followed by recompression using ultrabroadband chirped mirrors. The CEP of the driving pulses is stabilized using an experimental setup similar to that described by Baltuška et al. ${ }^{4}$, and the residual CEP fluctuations are about $0.1 \mathrm{rad}$ (r.m.s.). The CEP can be finely adjusted, without changing the pulse duration. The pulse polarization was then modulated with the above-mentioned technique. The XUV light is produced by focusing the polarization-modulated pulses onto an argon- or neon-gas jet created by a $400-\mu \mathrm{m}$-wide nozzle. The jet was positioned after the laser focus to select the short quantum trajectory by means of phase matching ${ }^{11,12}$. After pre-compensating for the plate's dispersion, we systematically checked that the pulses were unchirped in the interaction region. The radiation is collected and analysed by means of a high-throughput flat-field grazing-incidence spectrometer using an open solar-blind bidimensional detector ${ }^{24}$.

The experimental conditions were optimized to suppress any spectral broadening that was not directly connected to the confinement induced by the combined action of CEP and polarization gating (we used a moderate gas pressure and a low enough intensity to avoid spectral changes related to ionization). Experimentally, we checked that these effects were not significant because, without any gate (that is, with an intensity two times higher than with the gate), we observed well-defined plateau harmonics in argon and neon with a typical width of $\sim 1 \mathrm{eV}$.

\section{Received 17 December 2005; accepted 13 March 2006; published 23 April 2006.}

\section{References}

1. Corkum, P. B. Plasma perspective on strong-field multiphoton ionization. Phys. Rev. Lett. 71, 1994-1997 (1993).

2. Corkum, P. B., Burnett, N. H. \& Ivanov, M. Y. Subfemtosecond pulses. Opt. Lett. 19, 1870-1872 (1994).

3. Christov, I. P., Murnane, M. M. \& Kapteyn, H. C. High-harmonic generation of attosecond pulses in the single-cycle regime. Phys. Rev. Lett. 78, 1251-1254 (1997).

4. Baltuška, A. et al. Attosecond control of electronic process by intense light fields. Nature $\mathbf{4 2 1}$ 611-615 (2003).

5. Itatani, J. et al. Tomographic imaging of molecular orbitals. Nature 432, 867-871 (2004).
6. Nikura, H., Villeneuve, D. M. \& Corkum, P. B. Mapping attosecond electron wave packet motion. Phys. Rev. Lett. 94, 083003 (2005).

7. Lewenstein, M., Balcou, P., Ivanov, M. Y., L'Huillier, A. \& Corkum, P. B. Theory of high-harmonic generation by low-frequency laser fields. Phys. Rev. A 49, 2117-2132 (1994).

8. Farkas, G. \& Tóth, C. Proposal for attosecond light pulse generation using laser induced multiple-harmonic conversion processes in rare gases. Phys. Lett. A 168, 447-450 (1992).

9. Paul, P. M. et al. Observation of a train of attosecond pulses from high harmonic generation. Science 292, 1689-1692 (2001)

10. Mairesse, Y. et al. Attosecond synchronization of high-harmonic soft x-rays. Science 302, 1540-1543 (2003).

11. Antoine, P., L'Huillier, A. \& Lewenstein, M. Attosecond pulse trains using high-order harmonics. Phys. Rev. Lett. 77, 1234-1237 (1996).

12. Gaarde, M. B. \& Schafer, K. J. Space-time considerations in the phase locking of high harmonics. Phys. Rev. Lett. 89, 213901 (2002).

13. Kienberger, R. et al. Atomic transient recorder. Nature 427, 817-821 (2004).

14. Platonenko, V. T. \& Strelkov, V. Single attosecond soft-x-ray pulse generated with a limited laser beam. J. Opt. Soc. Am. B 16, 435-440 (1999).

15. Altucci, C. et al. Frequency-resolved time-gated high-order harmonics. Phys. Rev. A 58, 3934-3941 (1998).

16. Tcherbakoff, O. et al. Time gated high order harmonic generation. Phys. Rev. A 68, 043804 (2003).

17. Kovacev, M. et al. Temporal confinement of the harmonic emission through polarization gating. Eur. Phys. J. D 26, 79-82 (2003).

18. López-Martens, R. et al. Time-resolved ellipticity gating of high-order harmonic emission. Phys. Rev. A 69, 053811 (2004).

19. Chang, Z. Single attosecond pulse and xuv supercontinuum in the high-order harmonic plateau. Phys. Rev. A 70, 043802 (2004).

20. Strelkov, V. et al. Generation of attosecond pulses with ellipticity-modulated fundamental. Appl. Phys. B 78, 879-884 (2004).

21. Shan, B., Ghimire, S. \& Chang, Z. Generation of the attosecond extreme ultraviolet supercontinuum by a polarization gating. J. Mod. Opt. 52, 277-283 (2005).

22. Strelkov, V. et al. Single attosecond pulse production with an ellipticity-modulated driving IR pulse. J. Phys. B 38, L161-L167 (2005).

23. Nisoli, M. et al. Effects of carrier-envelope phase differences of few-optical-cycle light pulses in single-shot high-order-harmonic spectra. Phys. Rev. Lett. 91, 213905 (2003).

24. Poletto, L., Bonora, S., Pascolini, M. \& Villoresi, P. Instrumentation for analysis and utilization of extreme-ultraviolet and soft x-ray high-order harmonics. Rev. Sci. Instrum. 75, 4413-4418 (2004).

25. López-Martens, R. et al. Amplitude and phase control of attosecond light pulses. Phys. Rev. Lett. 94, 033001 (2005).

26. Kyung, T. K. et al. Single sub-50-attosecond pulse generation from chirp-compensated harmonic radiation using material dispersion. Phys. Rev. A 69, 051805 (2004).

27. Chang, Z. Chirp of a single attosecond pulse generated by a polarization gating. Phys. Rev. A 71, 023813 (2005).

28. Oron, D., Silberberg, Y., Dudovitch, N. \& Villeneuve, D. M. Efficient polarization gating of high-order harmonic generation by polarisation-shaped ultrashort pulses. Phys. Rev. A 72, 063816 (2006).

29. Seres, J. et al. Source of coherent kiloelectronvolt X-rays. Nature 433, 596 (2005).

30. Nisoli, M. et al. Generation of high energy $10 \mathrm{fs}$ pulses by a new pulse compression technique. Appl. Phys. Lett. 68, 2793-2795 (1996).

\section{Acknowledgements}

We acknowledge the support of the European community under LASERLAB-EUROPE Integrated Infrastructure Initiative Contract RII3-CT-2003-506350, and the European XTRA network (MRTN-CT-2003-505138), as well as the financial support from the Région Aquitaine, the Russian Science Support Foundation and the Italian MIUR PRIN project 2004029033

Correspondence and requests for materials should be addressed to E.C.

\section{Competing financial interests}

The authors declare that they have no competing financial interests.

Reprints and permission information is available online at http://npg.nature.com/reprintsandpermissions/ 